\title{
Neutrophil-lymphocyte ratio and platelet-lymphocyte ratio as an alternative to C-reactive protein in diagnostics of inflammatory state in patients with psoriasis
}

\author{
Aneta Gruchała', Aleksander Cisłak², Jacek Golański ${ }^{1}$
}

${ }^{1}$ Department of Haemostatic Disorders, Medical University of Lodz, Lodz, Poland, ${ }^{2}$ Institute of Applied Computer Science, Lodz University of Technology, Lodz, Poland

Corresponding author: Dr. Aneta Gruchała, E-mail: aneta.gruchala@umed.lodz.pl

\begin{abstract}
Background: The ratio of neutrophils to lymphocytes (NLR), the ratio of platelets to lymphocytes (PLR), the ratio of monocytes to lymphocytes (MLR), and mean platelet volume (MPV) are considered novel inflammatory markers. We stated a hypothesis that apart from C-reactive protein (CRP) level, also NLR, PLR, MLR, and MPV are higher in patients with psoriatic arthritis (PsA, where both skin and joints are involved) than in patients with psoriasis vulgaris (PsV). Material and Methods: Our study is based on a retrospective analysis. We collected laboratory data, namely CRP levels as well as a total blood count, and calculated the following additional parameters: NLR, PLR, MLR, and MPVLR (mean platelet volume/lymphocyte ratio). For all data, two groups of patients were compared: PsV with N=80 and PsA with $\mathrm{N}=80$. Results: CRP levels were significantly higher in PsA patients compared with those in PsV patients (median PsV - $19.43 \mathrm{nmol} / \mathrm{L}$ vs median PsA-37.90 nmol/L, p=0.001). Similarly, both NLR and PLR were higher in PsA patients than in PsV patients (NLR, median PsV - 2.0 vs median PsA - 2.28, p=0.030; PLR, median PsV - 111.61 vs median PsA - 121.85, $\mathrm{p}=0.027)$. Moreover, for patients with psoriatic arthritis a weak to moderate positive correlation between C-reactive protein levels and WBC, neutrophils count, monocytes count, platelets count, NLR, PLR, and MLR was observed. Conclusions: CRP levels are higher in patients with PsA than in patients with PsV, which can be helpful in predicting arthritis in patients with psoriasis. Furthermore, similar information can be obtained from a blood count. In particular, NLR and PLR are simple predictors which can indicate ongoing joint inflammation in patients with psoriasis, hence they can be used as an alternative to the CRP level.
\end{abstract}

Key words: Psoriasis; Psoriatic arthritis; Inflammation; Neutrophil-lymphocyte ratio; Platelet-lymphocyte ratio; Low-cost test

\section{INTRODUCTION}

Psoriasis is a chronic immune-mediated inflammatory disease which affects around $2-4 \%$ of the population [1]. Occurrence of psoriasis is believed to be associated with both environmental and genetic factors [2,3]. Psoriasis vulgaris (PsV), or chronic plaque psoriasis, is the most common type of psoriasis and affects approximately 90\% of patients with psoriasis. In psoriatic arthritis
(PsA), there is an inflammation of joints, usually coexisting with a cutaneous manifestation of psoriasis. PsA occurs in up to $30 \%$ of patients with psoriasis [4]. Psoriasis is frequently associated with significant comorbidities including diabetes mellitus, obesity, dyslipidemia, inflammatory bowel disease, psychiatric disorders, osteoporosis, and obstructive sleep apnea, as well as cardiovascular diseases, e.g. hypertension, myocardial infarction, and stroke [5-7].

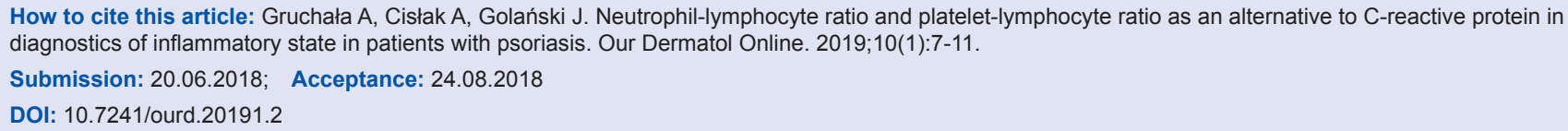


Multiple investigations were conducted with the goal of finding useful biomarkers within peripheral blood samples of patients with psoriasis and psoriatic arthritis. Unfortunately, they have not found a single, simple and clinically useful biomarker which could be specific for psoriasis [8]. On the other hand, C-reactive protein (CRP) level, which is a known inflammatory blood test marker of inflammation in the body, is elevated among psoriatic patients and can be used to assess disease severity and progression [9]. Recently, the ratio of neutrophils to lymphocytes (NLR), the ratio of platelets to lymphocytes (PLR), and the ratio of monocytes to lymphocytes (MLR) have been recognized as inflammatory markers [10-12]. NLR, PLR, and MLR are assessed as a prognostic of poor neoplastic disease outcome [13-17], whereas mean platelet volume (MPV) has been studied as an inflammatory marker in several diseases [18-20]. There are also a few studies which investigate simple markers, viz. NLR, PLR, and MPV in patients with psoriasis [21-24].

In this study, we analyzed, i.a., CRP level, NLR, PLR, MLR, and MPV in Polish patients with psoriasis. In particular, we put forward a hypothesis that patients with psoriatic arthritis have higher inflammatory biomarker levels than patients with psoriasis vulgaris.

\section{MATERIALS AND METHODS}

The study was carried out at the Department of Dermatology, Pediatric Dermatology and Oncology Clinic, Medical University of Lodz, Poland. It was a retrospective analysis, conducted for Polish patients with psoriasis vulgaris and psoriatic arthritis, hospitalized from January to December 2017. Inclusion criteria covered patients who were from 18 up to 90 years of age and diagnosed with psoriasis at least 1 year before the test was recorded. Exclusion criteria comprised a current or recent treatment with biological therapies, known inflammatory processes in the body (except psoriasis), and present history of a neoplastic disease. The study group consisted of 80 patients with psoriasis vulgaris (group 1) and 80 patients with psoriatic arthritis (group 2). All patients included in group 1 had present skin changes, and all patients included in group 2 had both skin and joints manifestations of the disease.

The following information was collected: C-reactive protein (CRP) level, red blood cells count (RBC), haemoglobin $(\mathrm{Hb})$, white blood cells count (WBC), lymphocytes, neutrophils, monocytes and platelets counts, and mean platelet volume (MPV). These blood morphology results (UniCel DxH 800 Coulter Cellular Analysis System, Beckman Coulter Inc, USA) and CRP concentration (particle-enhanced turbidimetric method, Cobas 6000 Analyzer, Roche, Germany) were obtained from the hospital laboratory. On the basis of blood morphology data, the following markers (indexes) were calculated: PLR (platelet/lymphocyte ratio), NLR (neutrophil/lymphocyte ratio), MLR (monocyte/lymphocyte ratio), and MPVLR (mean platelet volume/lymphocyte ratio).

Statistical analysis was performed with the SciPy (v. 1.1.0) suite for the Python programming language. The Shapiro-Wilk test was used to evaluate the normality of data distribution, and Brown-Forsythe test was employed to check for variance homogeneity. Continuous parameters are described using medians and the interquartile range, with the Mann-Whitney $\mathrm{U}$ test used for significance testing. The calculated sample size for a power of at least 0.8 and significance of at least 0.05 was equal to 80 patients in a group. The correlation between parameters was determined with the use of the Spearman coefficient.

\section{Ethics Statement}

The authors state no conflict of interest. Data used in this study was collected retrospectively and it was fully anonymous.

\section{RESULTS}

A total of 160 patients diagnosed with psoriasis - 80 patients with PsV (45 males, 35 females; mean age $48.48 \pm 16.08$ years; range $19-83$ years) and 80 patients with PsA (46 males, 34 females; mean age $49.45 \pm 13.74$ years; range $24-88$ years) were included in the study. Complete blood count data, C-reactive protein level, NLR, PLR, MLR, and MPVLR results are presented in Table 1. One can observe that CRP levels were significantly higher in PsA patients when compared with those in $\mathrm{Ps} V$ patients, and it was indeed the strongest predictor for the presence of arthritis in psoriasis patients. Moreover, both NLR and PLR were higher in PsA patients than in PsV patients. A statistically significant difference was also observed in terms of white blood cells, neutrophils and platelets counts, as well haemoglobin level between patients with psoriasis vulgaris and psoriatic arthritis. Let us 
Table 1: Marker comparison between patients with psoriasis vulgaris (PsV) and psoriatic arthritis (PsA)

\begin{tabular}{|c|c|c|c|}
\hline Parameter & PsV (N=80) Median (IQR) & PsA (N=80) Median (IQR) & p-value \\
\hline C-reactive protein [nmol/L] & $19.43(9.52-36.76)$ & $37.90(16.76-60.00)$ & 0.001 \\
\hline Red blood cells $\left[\times 10^{12} / L\right]$ & $4.67(4.31-5.00)$ & $4.71(4.35-5.12)$ & 0.231 \\
\hline Haemoglobin $[g / L]$ & $147.5(139.0-155.1)$ & $143.5(133.0-154.0)$ & 0.040 \\
\hline White blood cells $\left[\times 10^{9} / L\right]$ & $6.7(5.5-8.0)$ & $7.5(6.0-9.0)$ & 0.017 \\
\hline Lymphocytes [x 10\%/L] & $1.9(1.7-2.3)$ & $2.0(1.5-2.3)$ & 0.447 \\
\hline Neutrophils [x 10\%/L] & $3.85(2.79-4.58)$ & $4.35(3.20-5.75)$ & 0.016 \\
\hline Monocytes [x 10/L] & $0.6(0.5-0.7)$ & $0.6(0.5-0.8)$ & 0.084 \\
\hline Platelets $\left[\times 10^{9} / \mathrm{L}\right]$ & $212.5(192.4-259.5)$ & $233.5(197.4-298.0)$ & 0.034 \\
\hline Mean platelet volume [fL] & $9.25(8.54-9.86)$ & $9.30(8.54-10.10)$ & 0.242 \\
\hline Neutrophil to lymphocyte ratio & $2.00(1.43-2.49)$ & $2.28(1.61-3.23)$ & 0.030 \\
\hline Platelet to lymphocyte ratio & $111.61(91.45-138.45)$ & $121.85(93.87-164.38)$ & 0.027 \\
\hline Mean platelet volume to lymphocyte ratio & $4.87(3.89-5.66)$ & $4.81(3.97-5.81)$ & 0.418 \\
\hline Monocyte to lymphocyte ratio & $0.32(0.24-0.39)$ & $0.33(0.24-0.47)$ & 0.120 \\
\hline
\end{tabular}

Continuous data are presented as a median with the interquartile range (IQR, lower quartile-upper quartile) in parentheses. Data was not normally distributed and significance was analyzed using the Mann-Whitney $U$ test. P-value $<0.05$ was considered statistically significant (emphasized with the bold font).

also note that these differences between the two groups were in general less statistically significant for older groups of patients (e.g., born before 1970).

Other parameters, including RBC, lymphocytes, monocytes, MPV, MPVLR, and MLR were either similar or the difference between the two groups was not statistically significant. We also analyzed the Spearman correlation between the C-reactive protein level and other parameters, with results presented in Table 2. For PsA patients we observed a weak to moderate positive correlation with monocytes count $\left(\mathrm{r}_{\mathrm{s}}=0.238\right.$, $\mathrm{p}=0.033), \mathrm{WBC}\left(\mathrm{r}_{\mathrm{s}}=0.3, \mathrm{p}=0.007\right), \operatorname{MLR}\left(\mathrm{r}_{\mathrm{s}}=0.314\right.$, $\mathrm{p}=0.005)$, platelets count $\left(\mathrm{r}_{\mathrm{s}}=0.372, \mathrm{p}=0.001\right)$, neutrophils count $\left(r_{\mathrm{s}}=0.395, \mathrm{p}<0.001\right), P L R\left(\mathrm{r}_{\mathrm{s}}=0.395\right.$, $\mathrm{p}<0.001)$, and NLR $\left(\mathrm{r}_{\mathrm{s}}=0.439, \mathrm{p}<0.001\right)$. Hence, the highest correlation coefficient for patients with psoriatic arthritis was discovered for neutrophils count, PLR, and NLR. Furthermore, we received negative statistically significant correlation between CRP and haemoglobin levels, lymphocytes counts, and MPV for PsA patients. In patients with psoriasis vulgaris the correlation between the aforementioned CRP level and other parameters was generally not statistically significant, with the sole exception of platelets count.

\section{DISCUSSION}

We searched for promising markers which might be useful for the prediction of arthritis in psoriasis patients. Let us reiterate that serum C-reactive protein level is a popular inflammatory biomarker, which might be employed for this task. Besides CRP, there exist other markers in psoriatic patients such as IL-6, TNF- $\alpha$, E-selectin, or ICAM-1, however, they are costly to obtain and hence not commonly tested [25].
On the other hand, in this study we concentrated on parameters from routine blood tests and found that WBC, neutrophils, monocytes and platelets counts, NLR, as well as PLR were indeed higher for patients with PsA when compared to patients with PsV.

According to Solak et al. [21], both CRP level and NLR are promising predictors of inflammation in psoriasis (compared with the control group). In their study, PLR was not assessed and psoriatic arthritis was not considered. Polat et al. [22] evaluated CRP, NLR, and PLR in Turkish patients, which turned out to be significantly higher in people with PsV than in the control group. Kim et al. [23] compared parameters from routine blood tests in Korean patients with psoriasis vulgaris and psoriatic arthritis. Our results are in general consistent with their study, since they observed statistically significant differences in terms of CRP, WBC, neutrophils, platelets counts, NLR and PLR. Similarly to this work, both NLR and PLR were strong predictors for the presence of arthritis among psoriasis patients. On the other hand, Kim et al. reported monocytes counts to be significantly higher in PsA patients, a scenario which we did not observe. Asahina et al. [24] described a correlation between CRP and parameters including WBC, neutrophils, lymphocytes, platelets, MPV, NLR, and PLR in Japanese patients with psoriasis. Interestingly, they described significant correlations not only for PsA patients as in our study, but also for PsV patients. Canpolat et al. [26] investigated MPV in patients with psoriasis vulgaris, psoriatic arthritis, and healthy subjects. In their study, MPV was higher in patients with PsA than in patients with PsV, however, in our study MPV did not differ between the groups. In another note, mean platelet volume was regarded as 
www.odermatol.com

Table 2: Spearman correlation coefficient between C-reactive protein level and other parameters for patients with psoriasis vulgaris (PsV) and psoriatic arthritis (PsA)

\begin{tabular}{|c|c|c|c|c|c|c|}
\hline Parameter & rs PsV, $\mathrm{N}=80$ & 95\% Cl PsV & p-value PsV & rs $\mathrm{PsA}, \mathrm{N}=\mathbf{8 0}$ & 95\% CI PsA & p-value PsA \\
\hline White blood cells & 0.184 & {$[-0.038,0.388]$} & 0.103 & 0.300 & {$[0.086,0.487]$} & 0.007 \\
\hline Lymphocytes & 0.025 & {$[-0.196,0.243]$} & 0.826 & -0.241 & {$[-0.437,-0.022]$} & 0.031 \\
\hline Neutrophils & 0.177 & {$[-0.044,0.382]$} & 0.116 & 0.395 & {$[0.192,0.566]$} & $<0.001$ \\
\hline Monocytes & 0.104 & {$[-0.119,-0.316]$} & 0.359 & 0.238 & {$[0.019,0.435]$} & 0.033 \\
\hline Platelets & 0.230 & {$[0.010,0.428]$} & 0.041 & 0.372 & {$[0.166,0.547]$} & 0.001 \\
\hline Mean platelet volume & -0.169 & {$[-0.375,0.053]$} & 0.134 & -0.272 & {$[-0.464,-0.055]$} & 0.015 \\
\hline Neutrophil to lymphocyte ratio & 0.103 & {$[-0.119,0.316]$} & 0.363 & 0.439 & {$[0.242,0.600]$} & $<0.001$ \\
\hline Platelet to lymphocyte ratio & 0.211 & {$[-0.009,0.412]$} & 0.060 & 0.395 & {$[0.192,0.566]$} & $<0.001$ \\
\hline $\begin{array}{l}\text { Mean platelet volume } \\
\text { to lymphocyte ratio }\end{array}$ & -0.110 & {$[-0.322,0.113]$} & 0.332 & 0.129 & {$[-0.094,0.339]$} & 0.255 \\
\hline Monocyte to lymphocyte ratio & 0.135 & {$[-0.087,0.345]$} & 0.232 & 0.314 & {$[0.101,0.499]$} & 0.005 \\
\hline
\end{tabular}

Spearman correlation coefficient rs with a two-sided $95 \%$ confidence interval $(\mathrm{Cl})$. P-value $<0.05$ was considered statistically significant (emphasized with the bold font).

an inappropriate indicator of inflammation, which is again consistent with our results [27].

Let us also address the limitations of this study. The analysis was conducted retrospectively in a single university hospital. Even though we excluded patients who had a history of one or more significant inflammatory processes (except psoriasis), information regarding comorbidities might be incomplete, with a possible external influence on inflammatory biomarkers. Moreover, described parameters might be altered by many conditions which are difficult to control, such as dehydration or overhydration. We also did not consider skin lesions severity (which can be assessed, e.g. using Psoriasis Area and Severity Index).

\section{CONCLUSIONS}

Based on the obtained results, we regard CRP, NLR, and PLR as promising candidates for predictors of arthritis among patients with psoriasis. In particular, they were observed to be higher in patients with PsA than in patients with PsV. Hence, for the assessment of inflammation, the blood count might be sufficient, even without the need for a more complex CRP test. Moreover, higher white blood cell, neutrophils, or platelet counts might suggest a developing arthritis in people with psoriasis. These biomarkers are simple, easily accessible, and relatively inexpensive to obtain.

For achieving better results when it comes to psoriatic arthritis treatment, it is significant to begin the treatment early. Thanks to these aforementioned, easily obtainable biomarkers, chances to predict which patients with psoriasis are developing arthritis might be boosted. Furthermore, increased CRP level, NLR, and PLR indicate a greater inflammatory burden in patients with psoriatic arthritis compared to patients with psoriasis vulgaris. The elevation of inflammatory markers in patients with psoriasis could be associated with, for instance, increased cardiovascular risk. For this reason, patients with psoriasis who have high CRP level, NLR, or PLR, should be followed by a dermatologist more carefully, although more studies are required to show how these values could be used in clinical practice.

\section{Statement of Human and Animal Rights}

All procedures followed were in accordance with the ethical standards of the responsible committee on human experimentation (institutional and national) and with the Helsinki Declaration of 1975, as revised in 2008 .

\section{REFERENCES}

1. Parisi R, Symmons DP, Griffiths CE, Ashcroft DM. Global epidemiology of psoriasis: a systematic review of incidence and prevalence. J Invest Dermatol. 2013;133:377-85.

2. Herron MD, Hinckley M, Hoffman MS, Papenfuss J, Hansen $\mathrm{CB}$, Callis KP, et al. Impact of obesity and smoking on psoriasis presentation and management. Arch Dermatol. 2005;141:1527-34.

3. Gruchała A, Gawrońska M, Galica K, Kaszuba A. Genetyczne aspekty łuszczycy i łuszczycowego zapalenia stawów [in Polish; Genetic aspects of psoriasis and psoriatic arthritis]. Dermatol Prakt. 2018;2:35-40.

4. Zachariae H. Prevalence of joint disease in patients with psoriasis: implications for therapy. Am J Clin Dermatol. 2003;4:441-7.

5. Sanchez-Carazo JL, López-Estebaranz JL, Guisado C. Comorbidities and health-related quality of life in Spanish patients with moderate to severe psoriasis: a cross-sectional study (Arizona study). J Dermatol. 2014;41:673-8.

6. Xu T, Zhang YH. Association of psoriasis with stroke and myocardial infarction: meta-analysis of cohort studies. Br J Dermatol. 2012;167:1345-50.

7. Machado-Pinto J, Diniz Mdos S, Bavoso NC. Psoriasis: new comorbidities. An Bras Dermatol. 2016;91:8-14.

8. Villanova F, Di Meglio P, Nestle FO. Biomarkers in psoriasis and 
psoriatic arthritis. Ann Rheum Dis. 2013;72 Suppl 2:ii104-10.

9. Beygi S, Lajevardi V, Abedini R. C-reactive protein in psoriasis: a review of the literature. J Eur Acad Dermatol Venereol. 2014;28:700-11.

10. Balta S, Celik T, Mikhailidis DP, Ozturk C, Demirkol S, Aparci $\mathrm{M}$, et al. The relation between atherosclerosis and the neutrophillymphocyte ratio. Clin Appl Thromb Hemost. 2016;22:405-11.

11. Özer S, Yılmaz R, Sönmezgöz E, Karaaslan E, Taşkın S, Bütün İ, et al. Simple markers for subclinical inflammation in patients with Familial Mediterranean Fever. Med Sci Monit. 2015;21:298-303.

12. Ji H, Li Y, Fan Z, Zuo B, Jian X, Li L, et al. Monocyte/lymphocyte ratio predicts the severity of coronary artery disease: a syntax score assessment. BMC Cardiovasc Disord. 2017;17:90.

13. Janik S, Raunegger T, Hacker P, Ghanim B, Einwallner E, Müllauer $\mathrm{L}$, et al. Prognostic and diagnostic impact of fibrinogen, neutrophilto-lymphocyte ratio, and platelet-to-lymphocyte ratio on thymic epithelial tumors outcome. Oncotarget. 2018;9:21861-75.

14. Templeton AJ, McNamara MG, Šeruga B, Vera-Badillo FE, Aneja P, Ocaña A, et al. Prognostic role of neutrophil-to-lymphocyte ratio in solid tumors: a systematic review and meta-analysis. J Natl Cancer Inst. 2014;106:dju124.

15. Fan W, Zhang Y, Wang Y, Yao X, Yang J, Li J. Neutrophil-tolymphocyte and platelet-to-lymphocyte ratios as predictors of survival and metastasis for recurrent hepatocellular carcinoma after transarterial chemoembolization. PLoS One. 2015;10:e119312.

16. Zhou WJ, Wu J, Li XD, Wang Q, Ni XF, Jiang JT, et al. Effect of preoperative monocyte-lymphocyte ratio on prognosis of patients with resectable esophagogastric junction cancer. Zhonghua Zhong Liu Za Zhi. 2017;39:178-83.

17. Tanrikulu AC, Abakay A, Komek H, Abakay O. Prognostic value of the lymphocyte-to-monocyte ratio and other inflammatory markers in malignant pleural mesothelioma. Environ Health Prev Med. 2016;21:304-11.

18. Gasparyan AY, Ayvazyan L, Mikhailidis DP, Kitas GD. Mean platelet volume: a link between thrombosis and inflammation? Curr Pharm Des. 2011;17:47-58.

19. Şenel E, Acar B, Demir E. Mean Platelet Volume: A reliable marker of inflammation in recurrent apthous stomatitis and Behcet disease? Indian Dermatol Online J. 2017;8:468-70.

20. Safak S, Uslu AU, Serdal K, Turker T, Soner S, Lutfi A. Association between mean platelet volume levels and inflammation in SLE patients presented with arthritis. African Health Scien. 2014;14:919 24.

21. Solak B, Dikicier BS, Erdem T. Impact of elevated serum uric acid levels on systemic inflammation in patients with psoriasis. Angiology. 2017;68:266-70.

22. Polat M, Bugdayci G, Kaya H, Oğuzman H. Evaluation of neutrophil-to-lymphocyte ratio and platelet-to-lymphocyte ratio in Turkish patients with chronic plaque psoriasis. Acta Dermatovenerol Alp Pannonica Adriat. 2017;26:97-100.

23. Kim DS, Shin D, Lee MS, Kim HJ, Kim DY, Kim SM, et al. Assessments of neutrophil to lymphocyte ratio and platelet to lymphocyte ratio in Korean patients with psoriasis vulgaris and psoriatic arthritis. J Dermatol. 2016;43:305-10.

24. Asahina A, Kubo N, Umezawa $Y$, Honda H, Yanaba K, Nakagawa H. Neutrophil-lymphocyte ratio, platelet-lymphocyte ratio and mean platelet volume in Japanese patients with psoriasis and psoriatic arthritis: response to therapy with biologics. J Dermatol. 2017;44:1112-21

25. Dowlatshahi EA, van der Voort EA, Arends LR, Nijsten T. Markers of systemic inflammation in psoriasis: a systematic review and meta-analysis. Br J Dermatol. 2013;169:266-82.

26. Canpolat F, Akpinar H, Eskioğlu F. Mean platelet volume in psoriasis and psoriatic arthritis. Clin Rheumatol. 2010;29:325-8.

27. Leader A, Pereg D, Lishner M. Are platelet volume indices of clinical use? A multidisciplinary review. Ann Med. 2012;44:805-16.

Copyright by Aneta Gruchała, et al. This is an open access article distributed under the terms of the Creative Commons Attribution License, which permits unrestricted use, distribution, and reproduction in any medium, provided the original author and source are credited.

Source of Support: Nil, Conflict of Interest: None declared. 